\title{
Low Complexity Carrier Phase Estimation for 16-QAM Systems
}

\author{
Jilong $\mathrm{Han}^{1, \mathrm{a}}$, Wei $\mathrm{Li}^{1, \mathrm{~b}}$ and Haitao Yao ${ }^{1, \mathrm{c}}$ \\ ${ }^{1}$ Huazhong University of Science and Technology, Wuhan 430074, China; \\ a08hjl@163.com, bweilee@hust.edu.cn, ${ }^{\mathrm{c}} 793093788 @ q q . c o m$
}

Keywords: Coherent optical communication, carrier phase estimation, 16QAM.

\begin{abstract}
A low complexity carrier phase estimation (CPE) algorithm for 16-QAM systems is investigated in this paper. In the proposed CPE algorithm, the QPSK partitioning scheme is adopted to divide the symbols into three classes $\left(C_{1}, C_{2}\right.$ and $\left.C_{3}\right)$. The symbols in $C_{1}$ and $C_{3}$ are used to achieve the coarse estimation and then the symbols in $C_{2}$ are used to achieve the fine estimation. In addition, the $M$ th-power operation is replaced by the $M$-level absolute operation for the removal of modulated data phase, which greatly reduced the complexity. The simulation results show that the proposed algorithm has better linewidth tolerance that the traditional Viterbi and Viterbi (V\&V) algorithm.
\end{abstract}

\section{Introduction}

In the high-speed optical coherent systems, the digital signal processing (DSP) is the key technology to compensate the signal degradation. Carrier phase estimation (CPE) is an important technology used in optical coherent systems to compensate for the phase noise induced by the transmitter laser and the local oscillator (LO). For example, the Viterbi and Viterbi (V\&V) algorithm is widely in the quadrature phase shift keying (QPSK) systems to estimate the phase noise [1]. But for the 16-ary quadrature amplitude modulation (16QAM), the QPSK partitioning scheme is widely adopted to implement carrier phase estimation [2, 3].

In the DSP algorithm, the computational complexity is one of the very important factors to determine whether the algorithm can be implemented in practice. Recently, we proposed an improved $\mathrm{V} \& \mathrm{~V}$ algorithm which adopts the $M$-level absolute operation rather than the $M$ th-power operation for the removal of modulated data phase and effectively reduces the complexity [4].

In this paper, in order to further enhance the estimation accuracy, an improved carrier phase estimation (CPE) algorithm for 16-QAM systems is proposed. In this algorithm, the symbols in $C_{1}$ and $C_{3}$ are used to achieve the coarse estimation and then the symbols in $C_{2}$ are used to achieve the fine estimation. In addition, in order to reduce the complexity, the $M$-level absolute operation is also used to replace the $M$ th-power operation. By the simulation, we compare the proposed algorithm with the traditional V\&V algorithm and the modified CPE algorithm in Ref. [3]. The simulation results show that the proposed algorithm has both advantages of good linewidth tolerance and low computational complexity.

\section{Principle}

The modified CPE algorithm in Ref. [3] proposed a two-stage estimation algorithm, in which the first stage adopts the traditional $\mathrm{V} \& \mathrm{~V}$ algorithm with the symbols in $C_{3}$ to achieve the coarse estimation and the second stage with all the symbols to achieve the fine estimation (named $\mathrm{P}_{3}+\mathrm{MP}$ ). In the second stage, as shown in Fig. 1 (b)-(c), after the $4^{\text {th }}$-power operation, the symbols in $C_{2}$ are required to be rotated by a phase of $4 \theta_{\text {rot }}$ or $-4 \theta_{\text {rot }}$ according to the decision result of $C_{2}$ (if imag $\left\{\left(C_{2}\right)^{4}\right\}>0$, the rotation phase is $4 \theta_{\text {rot }}$, otherwise the rotation phase is $\left.-4 \theta_{\text {rot. }}\right)$. But, due to the interference of phase noise, the error decision of $C_{2}$ will occurred when the linewidth is large enough. To solve this problem, we propose an improved CPE algorithm for 16QAM systems.

As shown in Fig. 2, the QPSK partitioning scheme is adopted to divide the symbols into three classes $\left(C_{1}, C_{2}\right.$ and $\left.C_{3}\right)$. To reduce the complexity, we adopt the 4-level absolute operation rather 
than the $4^{\text {th }}$-power operation for the removal of modulated data phase [4]. After the removal of modulated data phase, the symbols in $C_{1}$ and $C_{3}$ are used to achieve the coarse phase estimation $\theta_{\text {est } 1}$. Then the symbols in $C_{2}$ are compensated by $\theta_{\text {est } 1}$ to reduce the phase noise and avoid the error decision. After rotating by a phase of $4 \theta_{\text {rot }}$ or $-4 \theta_{\text {rot }}$, we can achieve the fine estimation $\theta_{\text {est } 2 \text {. The final }}$ phase estimation value is $\theta_{\text {est }}=\left(\theta_{\text {est1 }}+\theta_{\text {est } 2}\right)$. After unwrapping, all the symbols are compensated.

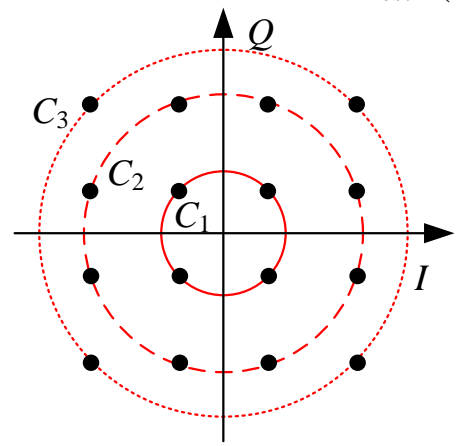

(a)

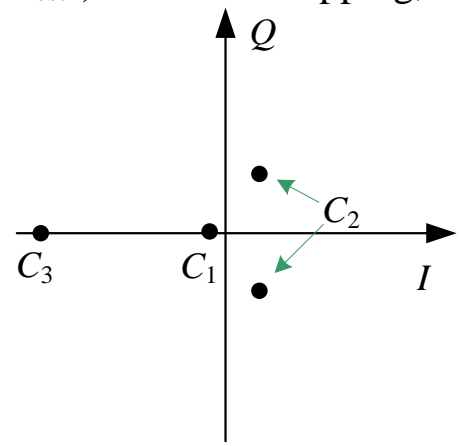

(b)

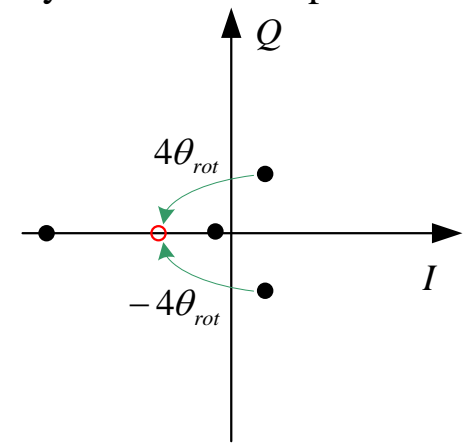

(c)

Fig. 1 Constellation of (a) ideal square 16-QAM, (b) after $4^{\text {th }}$-power operation, (c) after $4^{\text {th }}$-power and rotation operations

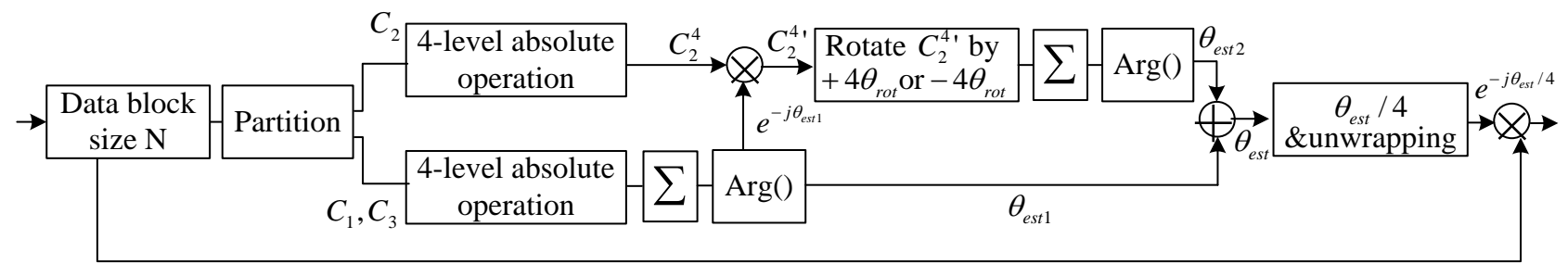

Fig. 2 The block diagram of the proposed CPE algorithm

\section{Simulation Results And Discussion}

In this section, the performance of the proposed algorithm is compared with that of the traditional $\mathrm{V} \& \mathrm{~V}$ and $\mathrm{P}_{3}+\mathrm{MP}$ algorithms in a 32-Gbaud square-16QAM coherent optical transmission system with single polarization. A pseudo-random binary sequence (PRBS) with a length of $2^{20}-1$ is used to obtain the bit error ratio (BER).

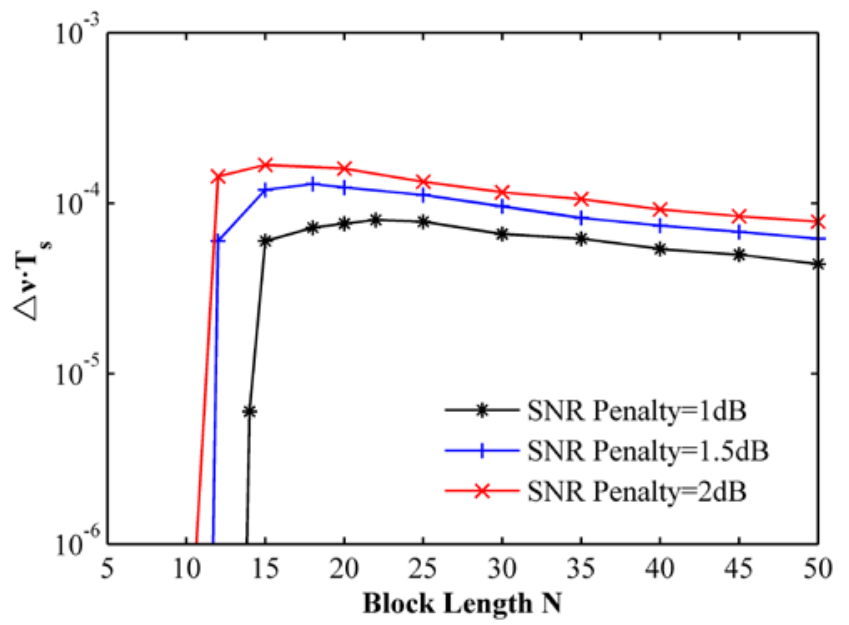

Fig. 3 Combined linewidth symbol duration product $\Delta v \cdot T_{S}$ versus block length $N$ for square-16QAM systems with different SNR penalties at BER of $10^{-3}$.

First, we investigate the optimal averaging block length $N$ of the proposed CPE algorithm. The differential encoding/decoding process is used in our simulations to avoid phase ambiguity. The reference SNR of square-16QAM is $17 \mathrm{~dB}$, which is obtained without any phase noise and CPE algorithm at $\mathrm{BER}=10^{-3}$. Fig. 3 shows the combined linewidth symbol duration product $\Delta v \cdot T_{s}$ over 
block length $N$ at the target BER of $10^{-3}$. From Fig. 3, we can see that the optimal block length $N$ of the proposed CPE algorithm is 22 at the SNR penalty of $1 \mathrm{~dB}$.

The optimal averaging block length $N$ of the $\mathrm{V} \& \mathrm{~V}$ algorithm is 35 [4] and the optimal averaging block lengths of the $\mathrm{P}_{3}+\mathrm{MP}$ algorithm are $N_{1}=50, N_{2}=30$ [3].

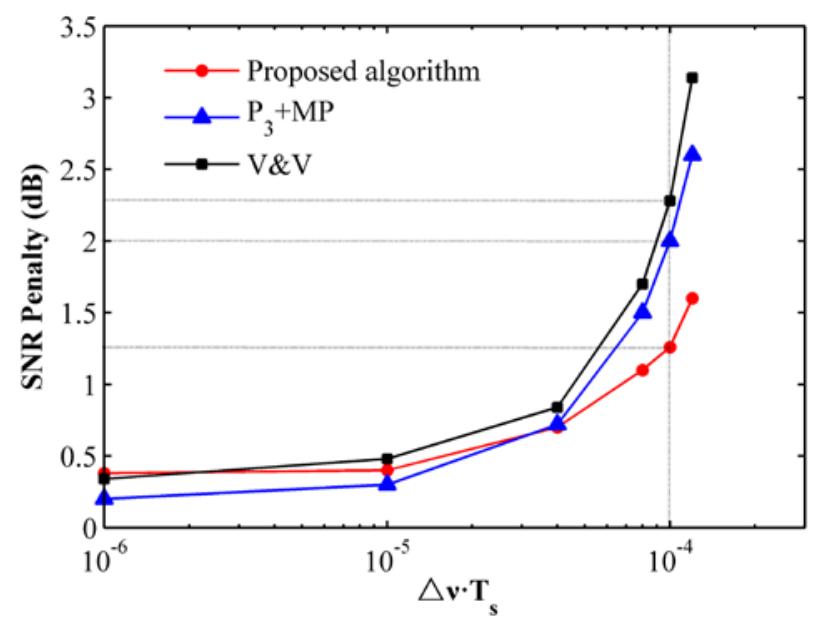

Fig. 4 SNR penalty at the target BER of $10^{-3}$ versus the combined linewidth symbol duration product $\Delta v \cdot T_{S}$

Next, we investigate the relationship between combined linewidth symbol duration product $\Delta v \cdot T_{S}$ and SNR penalty. As shown in Fig. 4, when the $\Delta v \cdot T_{S}$ is relatively smaller, the SNR penalties of the three CPE algorithms are very close. But along with increasing $\Delta v \cdot T_{s}$, an enhanced tolerance to linewidth is presented in the proposed CPE algorithm. When $\Delta v \cdot T_{S}=1 \times 10^{-4}$, the proposed algorithm reduces the SNR penalty by about $1 \mathrm{~dB}$ as compared with the $\mathrm{V} \& \mathrm{~V}$ algorithm.

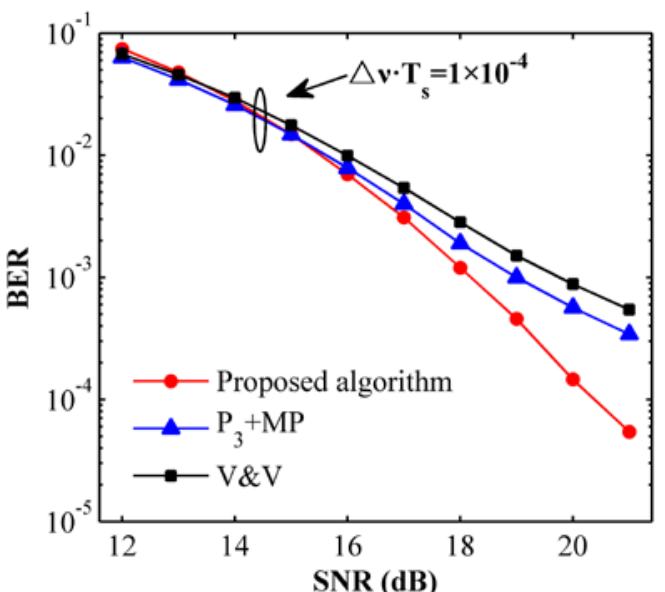

(a)

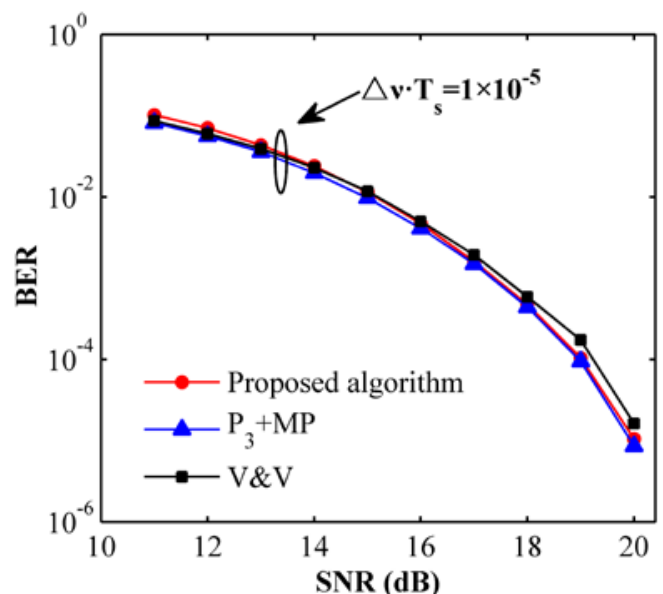

(b)

Fig. 5 BER versus SNR with (a) $\Delta v \cdot T_{S}=1 \times 10^{-4}$ and (b) $\Delta v \cdot T_{S}=1 \times 10^{-5}$

Fig. 5 shows the relationship between BER and SNR with different combined linewidth symbol duration product. When $\Delta v \cdot T_{S}=1 \times 10^{-5}$, the BER performance of the three CPE algorithms are very close. But when $\Delta v \cdot T_{S}=1 \times 10^{-4}$, along with increasing SNR, the proposed CPE algorithm presents a better BER performance than the $\mathrm{V} \& \mathrm{~V}$ and $\mathrm{P}_{3}+\mathrm{MP}$ algorithms.

\section{Complexity Analysis}

In this section, we compare the computational complexity of the proposed CPE algorithm, V\&V algorithm, and $\mathrm{P}_{3}+\mathrm{MP}$ algorithm. The computation is based on optimum implementations [5].

For the proposed CPE algorithm, to achieve the partition for $N$ symbols, it requires $2 N$ real multipliers, $N$ real adders, and $2 N$ comparators. The 4-level absolute operation requires $11 N$ real adders [4]. The summation and obtaining argument operations requires $N$-2 real adders and 1 look-up 
table (LUT). The coarse compensation to symbols in $C_{2}$ by $\theta_{\text {est1 }}$ and rotation operations requires $4 N$ real multipliers and $2 N$ real adders. In addition, the unwrapping operation requires 1 real adder and 1 comparator. Finally, the process of the phase compensation requires $4 N$ real multipliers, $2 N$ real adders, and 1 LUT.

The computational complexity of $\mathrm{V} \& \mathrm{~V}$ and $\mathrm{P}_{3}+\mathrm{MP}$ algorithms can be calculated by the similar methods and the detail process has been introduced in Ref. $[3,5]$. The complexity comparation of the three CPE algorithms is shown in Table 1. In addition, for clarity, the calculated values for the three CPE algorithms are shown in Table 2. Form Table 1 and Table 2, we can see that the complexity of the proposed algorithm is very low.

Table 1 Computational Complexity

\begin{tabular}{ccccc}
\hline Algorithm & Real Multipliers & Real Adders & Comparators & LUTs \\
\hline V\&V & $10 \mathrm{~N}+2$ & $5 \mathrm{~N}$ & $2 \mathrm{~N}+1$ & 1 \\
$\mathrm{P}_{3}+\mathrm{MP}$ & $7.5 \mathrm{~N}_{1}+12 \mathrm{~N}_{2}+2$ & $4 \mathrm{~N}_{1}+7 \mathrm{~N}_{2}$ & $\mathrm{~N}_{1}+\mathrm{N}_{2}+2$ & 2 \\
Proposed & $10 \mathrm{~N}+1$ & $18 \mathrm{~N}-2$ & $2 \mathrm{~N}+1$ & 3 \\
\hline \multicolumn{5}{c}{ Table 2 Calculated Computational Complexity } \\
\hline Algorithm & Real Multipliers & Real Adders & Comparators & LUTs \\
\hline V\&V & 352 & 175 & 71 & 1 \\
$\mathrm{P}_{3}+\mathrm{MP}$ & 737 & 410 & 82 & 2 \\
Proposed & 221 & 394 & 45 & 3 \\
\hline
\end{tabular}

\section{Summary}

In this paper, we proposed a CPE algorithm for 16QAM and investigate its performance. Through comparing the performance of the proposed algorithm, the $\mathrm{V} \& \mathrm{~V}$ algorithm, and the $\mathrm{P}_{3}+\mathrm{PM}$ algorithm, we find that the proposed algorithm has an enhanced tolerance to linewidth when the linewidth is relatively large. In addition, the complexity of the proposed algorithm is very low. Therefore, the proposed CPE algorithm is a preferable alternative for CPE in the 16QAM systems.

\section{References}

[1] A. J. Viterbi, and A. M. Viterbi, Nonlinear estimation of PSK-modulated carrier phase with application to burst digital transmission, IEEE Trans. Inform. Theory, Vol. 29 (1983) No. 4, p. 543-551.

[2] M. Seimetz, Laser linewidth limitations for optical systems with high-order modulation employing feed forward digital carrier phase estimation, in Proc. OFC, 2008, paper OTuM2

[3] K. P. Zhong, J. H. Ke, Y. Gao, et al., Linewidth-tolerant and low-complexity two-stage carrier phase estimation based on modified QPSK partitioning for dual-polarization 16-QAM systems, $J$. Lightw. Technol., Vol. 31 (2013) No. 1, pp. 50-57.

[4] J. Han, W. Li, Zhixue He, et al., A simplified implementation method of Mth-power for frequency offset estimation, IEEE Photon. Technol. Lett., Vol. 28 (2016) No. 20, pp. 2168-2171.

[5] J. H. Ke, K. P. Zhong, Y. Gao et al., Linewidth-tolerant and low-complexity two-stage carrier phase estimation for dual-polarization 16-QAM coherent optical fiber communications, J. Lightw. Technol., Vol. 30 (2012) No. 24, pp. 3987-3992. 